\title{
IMPORTÂNCIA DA REABSORÇÃO DO FERRO DA HEMORRAGIA INTESTINAL PROVOCADA PELA AÇĀO DOS VERMES NA PROGRESSÃO DA ANEMIA
}

Victorio Maspes *

Michiru Tamigaki *

RSPUB9/481

\begin{abstract}
MASPES, V. \& TAMIGAKI, M. Importância da reabsorção do ferro da hemorragia intestinal provocada pela ação dos vermes na progressão da anemia. Rev. Saúde públ., S. Paulo, 13:357-65, 1979.

RESUMO: Foram estudados 10 doentes, portadores de anemia ancilostomótica $e$ de grande parasitose. Foram determinados alguns parâmetros hematológicos como dosagem de hemoglobina, contagem de hemácias, hematócrito, volume corpuscular médio, hemoglobina corpuscular média, ferro sérico e siderofilina. Foram estudados o volume da perda de sangue intestinal, o ferro perdido nesta hemorragia e eliminado nas fezes, e o ferro reaborvido da hemorragia intestinal. Conclui-se que a reabsorção do ferro da hemoglobina por dia no tubo digestivo $\dot{e}$ de suma importância no retardamento da instalą̧áo da anemia ferropriva, causada pela parasitose ancilostomótica.
\end{abstract}

UNITERMos: Ancilostomose. Anemia ferropriva.

\section{NTROUCAO}

A anemia ancilostomótica é uma anemia ferropriva que se instala de modo insidioso em um organismo parasitado, e geralmente é decorrente de várias reinfecções. Esta é a conseqüência natural do meio ambiente em que vivem os indivíduos de baixa classe sócio-econômica e da zona rural. A anemia desenvolve-se lenta e progressivamente. embora a espoliação de sangue ocorra diariamente e em quantidades até consideráveis. Isto assim se estabelece porque parte do ferro da hemoglobina perdida pela hemorragia é reabsorvida e reaproveitada para a formação de novas hemácias $3, y_{1} 1$. .
Sem esta reabsorção, certamente a anemia seria mais grave e a sua instalação mais rápida, o que obrigaria o indivíduo a procurar o auxílio médico mais precocemente. Desse modo, até que os estoques de ferro do organismo sejam depletados, os doentes portadores de grande parasitose conseguem manter-se por periodo de tempo relativamente longo sem exibir qualquer sintomatologia.

Para demonstrar este importante aspecto da patogenia da anemia ancilostomótica, propusemo-nos a efetuar o presente trabalho. $O$ estudo foi realizado através da

\footnotetext{
* Do Serviço de Hematologia da Faculdade de Melicina da USP - Av. Dr. Arnaldo, 455 01246 - São Paulo, SP - Brasil.

* Do Departamento de Medicina Preventiva da Faculdade de Medicina da USP.
} 
MASPES, V. \& TAMIGAKI, M. Importância da reabsorção do ferro da hemorragia intestinal provocada pela ação dos vermes na progressão da anemia. Rev. Saúde públ., S. Paulo, 13: $357-65,1979$.

determinação da hematimetria, ferro sérico * siderofilina, perda de sangue intestinal, ferro da hemorragia perdido nas fezes, ferro da hemorragia intestinal reabsorvido, contagem de ovos e de vermes nas fezes. Estes dados possibilitaram a avaliação dos mecanismos que atuam favorecendo ou dif:cultando $D$ estabelecimento da anemia.

\section{MATERIAL E METODOS}

Foram estudados 10 doentes internados no Serviço de Hematologia do Hospital das Clínicas da Faculdade de Medicina da Universidade de São Paulo. Todos eram portadores de infectação ancilostomótica, comprovada em 2 exames parasitológicos de rotina. Todos os casos apresentaram grande parasitose, comprovada pela contagem de ovos nas fezes, que foi acima de 500.000 por dia e pelo número de vermes, superior a 400 . O critério observado para a classificação da grandeza de infecção foi a seguinte: pequena, até 100 vermes; média, de 100 a 250 vermes; grande, acima de 250 vermes. Foi incluido um caso, no qual se constatou esplenomegalia de 5 dedos, causada pela esquistossomose mansônica, cujos ovos foram identificados durante a contagem de ovos, e que não o haviam sido nos exames de rotina. Alguns casos eram também portadores de outras parasitoses como giardiase, ascaríase e tricuríase (Tabela 1). Não foram incluídos doentes portadores de outras patologias.

Determinação hematimétrica: A hemoglobina foi dosada pelo método da oxihemoglobina em meio alcalino; a contagem de hemácias foi feita em câmara; o hematócrito foi determinado pela técnica do microhematócrito; e para a dosagem do ferro sérico e da siderofilina foi utilizado o método de Ramsay modificado ${ }^{12}$.

Através da utilização de métodos radioisotópicos ${ }^{11.14 .15}$, determinou-se a perda de sangue intestinal por dia, e calculou-se a perda efetiva de ferro e a quantidade de ferro reabsorvida.
A determinação da quantidade de sangue perdido nas fezes obedeceu aos seguintes passos: as hemácias do paciente foram marcadas com o radiocromato de sódio $(" 1 \mathrm{Cr})$, acrescentando-se o material radioativo, em doses adequadas, a $20 \mathrm{ml}$ de sangue venoso colhido com anticoagulante. Após $30 \mathrm{~min}$. de incubação a $37^{\circ} \mathrm{C}$, o sangue marcado era reinjetado no paciente. $\mathrm{O}$ radioferro ("9 $\mathrm{Fe}$ ) foi injetado em doses adequadas 7 dias antes do radiocromo. F.ste período de tempo, em pacientes ferropênicos, é suficiente para que se verifique a incorporação total do ferro à hemoglobina. A técnica utilizada para estas duas marcações foi por nós descrita anteriormente ${ }^{11}$. Dois dias após a marcação com o radiocromo, iniciava-se a coleta de fezes, administrandose ao paciente 2 gramas de carmim, em cápsulas gelatinosas. O doente era submetido a um regime alimentar pobre em resíduos, e tomava $1 \mathrm{~g}$ de cáscara sagrada à noite, a fim de evitar a constipação intestinal $e$ o endurecimento das fezes. Após a ingestão do carmim, as fezes eram colhidas em comadre, a fim de observar a sua coloração, e tão logo se tornassem avermelhadas, eram colocadas em um pote com capacidade de $2 \mathrm{~kg}$, previamente tarado; todas as fezes das evacuações seguintes eram coletadas nesse pote durante 4 a 6 dias; no 4", 5" ou 6" dia do início da marcação das fezes, era dado novamente carmim ao doente, na mesma quantidade e horário anteriores, e as fezes eram coletadas em comadre, sendo recolhidas no pote até que se tornassem avermelhadas; quando isto ocorresse, as fezes eram desprezadas, indicando o término da coleta das fezes. Finalmente, o pote era pesado. a fim de se calcular a quantidade total das fezes nos 4,5 ou 6 dias. 0 material era submetido à homogeneização, e uma amostra de aproximadamente $4 \mathrm{~g}$ de fezes era colocada em um tubo de contagem previamente tarado. O tubo era pesado com as fezes para se obter o peso da amostra; em seguida, era centrifugado para se juntar as fezes no fundo do tubo $e$ 
MASPES, V. \& TAMIGAKI, M. Importância da reabsorçâo do ferro da hemorragia intestinal provocada pela ação dos vermes na progressão da anemia. Rev. Saüle pubt., S. Pauio, 13: $357-65,1979$.

equalizar a geometria das amostras. Uma amostra de sangue era colhida com anticoagulante $24 \mathrm{~h}$ antes da ingestão do carmim, e mais 3 amostras durante o periodo da colheita de fezes. As amostras de sangue eram lisadas com saponina, e uma aliquota de $4 \mathrm{ml}$ desses lisados era colocada em tubo de contagem. Os 4 tubos eram contados no cintilador de poço, para a medida da radioatividade, no mesmo dia da contagem das amostras de fezes.

Através das determinaçōes da atividade radioativa das amostras de sangue, era calculado o decaimento médio, a fim de se obter a média das atividades do $51 \mathrm{Cr}$ e do ${ }^{59} \mathrm{Fe}$ no sangue durante a colheita de fezes. Sabendo-se que o cromo injetado e perdido das fezes não é reabsorvido $:, 3,4$, calculou-se o volume de sangue total perdido nas fezes por dia, com a aplicação da seguinte fórmula:

$$
\text { (1.) } \quad S p=\frac{31 \operatorname{Crf} \times P}{31 \operatorname{Crs}}
$$

Onde:

$S p$ é o sangue perdido nas fezes por dia em mililitros

¿l Crf é o número de c.p.m. de ${ }^{11} \mathrm{Cr}$ por grama de fezes

$P$ é o peso médio das fezes de 24 horas

${ }^{5} 1$ Crs é o número de c.p.m. por mililitro de sangue

A quantidade total de hemoglobina perdida por dia pela via digestiva será:

$$
\text { (2.) } \mathrm{Hp}=\frac{\mathrm{Hb} \times \mathrm{Sp}}{100}
$$

Onde:

$H p$ é a hemoglobina total perdida pela via digestiva em gramas

$H b$ é a concentração de hemoglobina no sangue em $\mathrm{g} / \mathrm{dl}$
O ferro total da hemoglobina perdida por dia pela via digestiva foi calculado pela seguinte fórmula:

$$
\text { (3.) Hfe }=\mathrm{Hp} \times 3,35
$$

Onde:

Hfe é o ferro total da hemoglobina perdida por dia pela via digestiva

3,35 é o teor de ferro em $m g$ por grama de hemoglobina.

A atividade do ${ }^{5} 9 \mathrm{Fe}$ por grama de hemoglobina no sangue foi calculado pela fórmula :

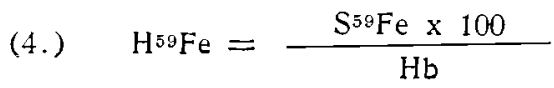

Onde:

$\mathrm{H}^{59} \mathrm{Fe}$ é o número de c.p.m. do ${ }^{59} \mathrm{Fe}$ por grama de hemoglobina

$S^{59} \mathrm{Fe}$ é o número de c.p.m. do ${ }^{59} \mathrm{Fe}$ por $\mathrm{ml}$ de sangue

A atividade radioativa do ferro nas fezes de 24 horas será:

(5.) ${ }^{59}$ Fet $={ }^{59}$ Fef $\times P$

Onde :

${ }^{59}$ Fet é o número de c.p.m. do ${ }^{59} \mathrm{Fe}$ por grama de fezes

${ }^{59}$ Fef é o número de c.p.m. do ${ }^{39} \mathrm{Fe}$ nas fezes de 24 horas

Calculou-se a atividade radioativa do ferro da hemoglobina total perdida por dia nas fezes através da seguinte fórmula.

(6.) $M^{59 \mathrm{Fet}}=\mathrm{H}^{5 \mathrm{~s} \mathrm{Fe}} \times \mathrm{Hp}$

Onde:

$H^{5}{ }^{\circ} F$ et significa o número de c.p.m. do ${ }^{59} \mathrm{Fe}$ total da hemoglobina perdida por dia. 
MASPES, V. \& TAMIGAKI, M. Importancia da reabsorção do ferro da hemorragia intestinal provocada pela ação dos vermes na progressão da anemia. Rev. Saúde públ., S. Paulo, 13: $357-65,1979$.

Portanto, a percentagem do ferro da hemoglobina perdida e eliminada com as fezes de 24 h é calculada como se segue:

$$
\mathrm{Fe} \%=\frac{{ }^{59 \mathrm{Fet}} \times 100}{\mathrm{H}^{50 \mathrm{Fet}}}
$$

Finalmente, o ferro da hemoglobina perdido e reabsorvido por dia, será:

$$
\text { (8.) } \quad \text { Fer }=\frac{\text { Hfe } \times(100-\mathrm{Fe} \%)}{100}
$$

Onde:

Fer é o ferro hemoglobinico reabsorvido em $\mathrm{mg}$ por dia.

E a percentagem do terro henoglobinico reabsorvido por dia, será:

$$
\text { Fer } \%=100-\mathrm{Fe} \%
$$

A contagem de ovos nas fezes no periodo de $24 \mathrm{~h}$, seguiu a técnica de Ferreira e col. ${ }^{6,7}$.

A contagem de vermes nas fezes foi efetuada (após administração de vermifugo) utilizando-sẹ o método de Amato e col. 1 .

\section{RESULTADOS}

A idade dos doentes mostrou-se entre 10 e 30 anos. Quatro casos pertenciam ao sexo masculino e os demais, ao sexo feminino. $O$ peso corporal apresentou-se de 25 a 59 $\mathrm{kg}$. Todos os doentes pertenciam à baixa condição sócio-econômica, conforme a especificação que consta na Tabela 1. Um dos casos apresentava regular condição alimentar, enquanto dois casos referiam boas condições de alimentação, de acordo com condições de alimentação de acordo com a especificacão referida na Tabela 1.0 período de exposição à parasitose era longo, sendo variável de 10 a 26 anos, durante o qual, progressivamente, os pacientes adquiriram os vermes até se tornarem intensamente parasitados. Seis destes doentes referiam estar expostos à parasitose durante toda a vida e os demais casos, durante boa parte da vida. Quanto à duraçãn dos sintomas de anemia, variou de 3 a 60 meses em 9 casos, e o caso restante queixou-se de que sempre foi doente. Neste caso, foi constatada uma esplenomegalia de 5 dedos, em conseqüência da esquistossomose mansônica concomitante. Estes dados são mostrados na Tabela 1 .

O quadro hematológico apresentou-se semelhante em todos os casos estudados, com o hematócrito variável de 7 a $32 \%$, a hemoglobina de $1,8 \mathrm{~g} / \mathrm{dl}$ a $8,2 \mathrm{~g} / \mathrm{dl}$, e o número de hemácias de $0,9 \times 10^{6} / \mathrm{mm}^{3}$ a $3,7 \times 10^{6} / \mathrm{mm}^{3}$, esta última contagem efetuada em 8 casos. Quanto ao volume corpuscular médio, calculado em 8 casos, mostrou-se variável de 64 a $95 \mathrm{u}^{3}$. A hemoglobina corpuscular média, nesses 8 casos, mostrou uma variação de 16 a 29 pg (Tabela 2).

O ferro sẻrico apresentou-se variável de 18 a $52 \mathrm{ug} / \mathrm{dl}$, nos 10 casos do estudo, enquanto a siderofilina total mostrou-se variável de 312 a $442 \mathrm{ug} / \mathrm{dl}$, em 7 casos. (Tabela 2).

Quanto ao volume de sangue perdido pela hemorragia intestinal, ocorreu uma variação de $18,6 \mathrm{ml}$ a $87,4 \mathrm{ml}$ por dia em todos os casos, e o cálculo da hemoglobina perdida na hemorragia demonstrou uma variação de 1,2 a 5,4 g por dia (Tabela 3).

O ferro perdido pela hemorragia intestinal mostrou uma variação de 3,9 a 18,1 $\mathrm{mg} / \mathrm{dia}$, enquanto o ferro reabsorvido foi variável de zero a $11,9 \mathrm{mg} / \mathrm{dia}$, que corresponde à percentagem de $66 \%$ de reabsorção. Assim, o ferro efetivamente perdido nas fezes foi no mínimo de $1,7 \mathrm{mg} /$ dia e no máximo de $6,9 \mathrm{mg} /$ dia (Tabela 3 ). 
MASPES, V. \& TAMIGAKI, M. Importancia da reabsorção do ferro da hemorragia intestinal provocada pela ação dos vermes na progressão da anemia, Rev. Saúde pübl., S. Paulo, 13: 357-65, 1979.

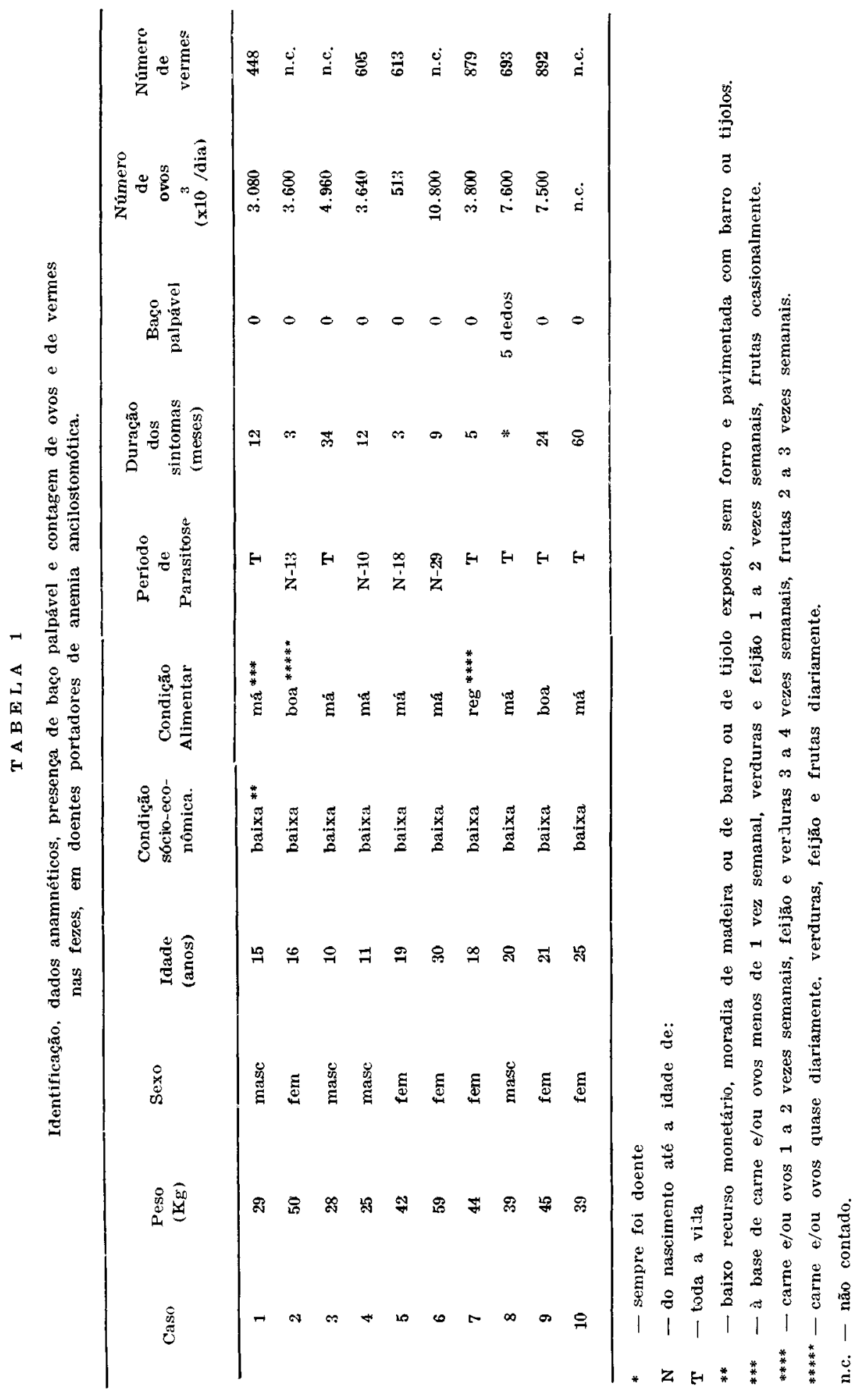


MASPES, V. \& TAMIGAKI, M. Importância da reabsorção do ferro da hemorragia intestinal provocada pela ação dos vermes na progressão da anemia. Rev. Saúde públ., S. Paulo, 13: $357-65,1979$.

T A B E L A 2

Hematmetria, ferro sérico e siderofilina total no primeiro atendimento ambulatorial. em doentes portadores de anemia ancilostomótica.

\begin{tabular}{|c|c|c|c|c|c|c|c|}
\hline Caso & $\begin{array}{c}\text { Hemato- } \\
\text { crito } \\
(\%)\end{array}$ & $\begin{array}{l}\text { Henroglo- } \\
\text { binat } \\
(\mathrm{g} / 100 \mathrm{ml})\end{array}$ & $\begin{array}{l}\text { Hemácids } \\
\left(\times 10^{6} \mathrm{~mm} 3\right)\end{array}$ & $\begin{array}{c}\text { V.C.M. } \\
\text { (us) }\end{array}$ & $\begin{array}{c}\text { H.C.M. } \\
(p g)\end{array}$ & $\begin{array}{c}\text { Ferro } \\
\text { sérico } \\
\text { (ug/10o } \\
\text { ml) }\end{array}$ & $\begin{array}{l}\text { Sitero- } \\
\text { filina total } \\
\text { (ug/100 } \\
\text { ml) }\end{array}$ \\
\hline$I$ & 8 & 2.6 & 0,9 & 88 & 28 & 52 & 340 \\
\hline 2 & 18 & 4,2 & 1,9 & 95 & 26 & 42 & 420 \\
\hline 3 & 15 & $: 3,5$ & 1.7 & 88 & 20 & 21 & 360 \\
\hline 4 & 32 & 8.2 & 3,5 & 94 & 29 & 38 & 359 \\
\hline 5 & 22 & 6,6 & 2,3 & 95 & 26 & 39 & n.d. \\
\hline 6 & 19 & 6.0 & n.d. & n.d. & n.d. & 18 & $n, d$. \\
\hline 7 & 22 & 5,4 & 2,4 & 91 & 22 & 32 & 312 \\
\hline 8 & 7 & 1,8 & 11.9 & 77 & 20 & 22 & $4: 2$ \\
\hline 9 & 14 & 2,8 & נl. & n.d. & n.d. & 19 & n.d. \\
\hline 10 & 24 & 6.4 & 3,7 & 64 & 16 & 18 & 442 \\
\hline
\end{tabular}

T A B E L A :;

Volume de sangue. hemoglobina e ferro perdidos pela hemorragia intestinal, ferro reabsorvida da hemorragia intestinal e ferro efetivamente perdido nas fezes, em doentes portadores de anemia ancilostomótica.

\begin{tabular}{ccccccc}
\hline Caso & $\begin{array}{c}\text { Volume de sall- } \\
\text { gue perdido pela } \\
\text { hemorragia } \\
\text { intestinal } \\
\text { (ml/dia) }\end{array}$ & $\begin{array}{c}\text { Hemoglobina } \\
\text { perdida pela } \\
\text { hemorragia } \\
\text { intestinal } \\
\text { (g/dia) }\end{array}$ & $\begin{array}{c}\text { Ferro per- } \\
\text { dido pela } \\
\text { hemorragia } \\
\text { intestinal } \\
\text { (mg/dia) }\end{array}$ & $\begin{array}{c}\text { Ferro reabsorvido } \\
\text { da hemorragia } \\
\text { intestinal } \\
\text { (mg/dia) }\end{array}$ & $\begin{array}{c}\text { Ferro da hemor- } \\
\text { ragia efetivamen- } \\
\text { te }\end{array}$ & $\begin{array}{c}\text { perdido nas } \\
\text { fezes } \\
\text { (mg/dia) }\end{array}$ \\
\hline 1 & 27,8 & 1,4 & 4,6 & 2,9 & 64 & 1,7 \\
2 & 44.7 & 4,1 & 13,6 & 6,7 & 49 & 6,9 \\
3 & 87,4 & 5,4 & 18,1 & 11,9 & 66 & 6,2 \\
4 & 23,7 & 2,0 & 6,5 & 4,4 & 63 & 2.1 \\
5 & 18,6 & 1,2 & 3,9 & 1,6 & 42 & 2,3 \\
6 & 65,7 & 3,1 & 10,5 & 4,8 & 46 & 5,7 \\
7 & 25.2 & 2,5 & 8,4 & 4,2 & 50 & 4,2 \\
8 & 42,7 & 1,8 & 6,0 & 2,3 & 39 & 3,7 \\
9 & 42,7 & 1,6 & 5,4 & 0,0 & 0 & 5,4 \\
10 & 29,0 & 1,8 & 6,0 & 2,9 & 48 & 3.1 \\
\hline
\end{tabular}


MASPES, V. \& TAMIGAKI, M. Importância da reabsorção do ferro da hemorragia intestinal provocada pela ação dos vermes na progressão da anemia. Rev. Saúde públ., S. Paulo, 13: $357-65,1979$.

\section{DISCUSSAO}

E grande a repercussão, no organismo, de uma hemorragia intestinal crônica, que ocorre em pequenas mas constantes quantidades, como acontece nos individuos portadores de ancilostomose. O metabolismo peculiar do ferro e a patogenia da ancilostomose permitem compreender como surge - principal efeito da espoliação de sangue pelos vermes.

Uma pequena perda de sangue para o meio exterior é seguida de equivalente absorção de ferro alimentar, sem qualquer repercussão grave para o organismo. Entretanto, se essa perda se mantiver durante muito tempo, por meses ou anos, o ferro alimentar, cuja disponibilidade e absorção é limitada, não conseguirá manter os parâmetros hematimétricos em niveis normais. O organismo lança mão, então, dos depósitos desse metal para essa situação. Temporariamente, haverá equilibrio entre o ferro perdido e o utilizado pela medula óssea na formação de hemoglobina. O rompimento desse equilíbrio surgirá quando os depósitos de ferro forem depletados. A medida que os estoques de ferro forem esgotados, os glóbulos formados conterāo menor quantidade de hemoglobina, ao lado de alterações na sua forma. Estabelece-se, então, a anemia, que se manifesta no organismo espoliado de ferro, ao qual se superpõe o componente hemolítico, decorrente da malformação das hemácias. O tempo que demanda todo esse processo, desde o início da espoliação até o estabelecimento da anemia, é relativamente longo, na dependência do volume de sangue perdido, da grandeza dos depósitos de ferro e também da disponibilidade e da capacidade de absorção do ferro alimentar. Como a hemorragia ocorre pela via intestinal, parte do ferro hemoglobínico será reabsorvido pela mucosa intestinal, e assim a instalação da anemia será mais tardia do que em relação a outras vias de sangramento, que não permitem a reabsorção e a reutilização de parte do ferro da hemoglobina contida no volume de sangue perdido. A anemia ancilostomótica ocorre justamente através desse mecanismo, que envolve a reabsorção do ferro perdido pela mucosa intestinal $5,8,13$. Conrad $^{3}$ e Layrisse 8 demonstraram que 0 ferro da hemoglobina é mais absorvível que - ferro alimentar ou o inorgânico, pelo que é fácil deduzir que a perda intestinal efetiva de ferro, nesses casos, é de menor magnitude que a contida no volume de sangue perdido, através da sucção do verme.

Nos 10 casos deste estudo, a perda hemorrágica intestinal variou de 18,6 a $87,4 \mathrm{ml}$ por dia, o que corresponde à perda hemoglobínica de $1,2 \mathrm{~g}$ a $5,4 \mathrm{~g}$ por dia, ou de $3,9 \mathrm{mg}$ a $18,1 \mathrm{mg}$ de ferro hemoglobínico por dia. Se o doente realmente perdesse essa quantidade de ferro hemoglobínico, em poucos dias teria sido espoliado de tal modo a apresentar falência total do setor eritrocitário, mesmo sob produção máxima medular. Mas, na verdade, parte do ferro perdido na hemorragia provocada pelo verme foi reabsorvido, como se mostra neste estudo. Verificou-se a reabsorção do ferro hemoglobínico da hemorragia em niveis de até $11,9 \mathrm{mg}$ por dia, - que corresponde a $66 \%$ do ferro da hemorragia. Esses dados indicam que a perda efetiva de ferro foi de $1,7 \mathrm{mg}$ por dia, no minimo, e de $6,9 \mathrm{mg}$ por dia, no máximo.

Todos esses resultados são dependentes também de um fator importante, que consiste na influência do tipo de alimentação sobre a absorção de ferro, tanto orgânico quanto inorgânico ${ }^{10}$. A alimentação da população rural, à qual pertencem os casos deste estudo, baseia-se em farináceos e em leguminosos, e somente ocasionalmente a carne toma parte nas refeições desses indivíduos. Sabe-se que a carne, além de ser a maior fonte de ferro absorvivel, fornece proteínas degradadas que facilitam sua absorção, enquanto que os farináceos dificultam esse processo. E sabido que o ferro hemoglobínico é mais absorvível do que as outras formas de ferro 3.8 , o que favorece 
MASPES, V, \& TAMIGAKI, M. Importância da reabsorção do ferro da hemorragia intestinal provocada pela ação dos vermes na progressão da anemia, Rev. Saúde públ., S. Paulo, 13: 357-65, 1979.

a evolução de uma anemia causada por hemorragia crônica intestinal.

Após analisar os dados desta investigação, depreende-se a importância da reabsorção do ferro pela mucosa intestinal na progressão da anemia, sem o que haveria certamente a rápida instalação do processo.

Todos os doentes apresentaram grande parasitose, pois estavam expostos à infecção pelo parasita durante quase toda sua vida. Isto determinou a grande espoliação de sangue verificada nos doentes, mas os sintomas de anemia surgiram após tempo relativamente longo, isto $\epsilon$, a anemia estabeleceu-se muito tempo após o inicio da parasitose. Um doente portador de grande parasitose pode, pois, viver normalmente durante prolongado periodo de tempo antes de surgirem os sintomas de anemia, graças ao ferro alimentar, ao seu depósito de ferro e à reabsorção de ferro da hemorragia provocada pelo verme.

\section{CONCLUSöES}

1) Uma grande parasitose ancilostomótica invariavelmente conduz o indivíduo a um estado de grave anemia. O tempo que demanda para o estabelecimento desta anemia, tipo ferropriva, é relativamente longo, embora as perdas de hemoglobina pelas fezes sejam consideráveis (da ordem de 1 a $5 \mathrm{~g} / \mathrm{dia})$.

2) Os fatores que influenciam a absorção do ferro alimentar, o tipo de alimentação, bem como os depósitos do organismo, são elementos importantes na instalação da anenia ancilostomótica. Entretanto, a reabsorção do ferro da hemorragia intestinal, que ocorre nesta parasitose, mostrou ser de relevante importância na fisiopatologia da ancilostomose.

3) O ferro perdido pela hemorragıa intestinal variou de $3,9 \mathrm{mg} /$ dia até 18,1 $\mathrm{mg} / \mathrm{dia}$, e o ferro reabsorvido dessa hemorragia variou de $0,0 \mathrm{mg} / \mathrm{dia}$ a $11,9 \mathrm{mg} / \mathrm{dia}$. $O$ ferro efetivamente perdido foi portanto menor que o da hemorragia, isto é, no minimu de $1,7 \mathrm{mg} / \mathrm{dia}$ e no máximo de 6,9 $m g / d i a$.

4) Devido à reabsorção do ferro da hemorragia intestinal, uma grande parasitose no indivíduo determina anemia de modo insidioso, em tempo mais longo que a hemorragia crônica extra-intestinal. Esta ultima evolui grave e rapidamente, pois não apresenta o componente de reaborção do ferro da hemorragia.

MASPES, V. \& TAMIGAKI, M. IThe importance of iron reabsorption in intestinal hemorrhage caused by worms in the course of anemial Rev. Saúde públ., S. Paulo, $13: 357-65,1979$.

ABSTRACT: Ten patients presenting ancylostomotic anemia and a high rate of parasitosis were submitted to several hematologic determinations: hemoglobin level, erythrocyte count, hematocrit, mean corpuscular volume, mean corpuscular hemoglobin, serum iron level, and transferrin. In addition, measurements were made of the blood volume lost by intestinal bleeding, of iron loss through hemorrhage and in feus, and of iron reabsorption from intestinal bleeding. The main reason for the late appearance of iron deficiency anemia in ancylostomiasis is the high degree of dally iron absorption in the digestive tract, originating from hemoglobin breakdown.

UNITERMS: Ancylostomiasis. Anemia, hypochromic. 
MASPES, V. \& TAMIGAKI, M. Importância da reabsorção do ferro da hemorragia intestinal provocada pela ação dos vermes na progressão da anemia. Rev. Saúde públ., S. Paulo, 13: $357-65,1979$.

\section{REFERENCIAS BIBLIOGRAFICAS}

1. AMATO NETO, V. et al. Diagnóstico das parasitoses intestinais pelo exame de fezes. 3a. ed. São Paulo, Atheneu Editora, 1969 .

2. CLARK, C. $\mathrm{H}$. et al. A quantitatıve measurements of the blood loss caused by Ancylostomyasis in dogs. Amer. $J$. vet. Med., 22:370-3, 1961.

3. CONRAD, M. E. et al. Human absorption of hemoglobin iron. Gastroenterology, 53:5-10, 1967.

4. EBAUGH, F. G. et al. Quantitative measurements of gastrointestinal blood loss. Amer. J. Med., 25:159-81, 1958.

5. FARID, $Z$, et al. Iron loss and reabsorption in Ancylostoma duodenale infection and bilharzial caloric polyposis. Trans. roy. Soc, trop. Med. Hyg., 64:881-4 1970 .

6. FERrEIRA, C. S. Contagem de ovos de heimintos nas fezes: algumas modificações. Rev. paul. Med., 68:240, 1966.

7. FERREIRA, C. S. \& REIS, A. A. Contagem de ovos de helmintos nas fezes; com. paração entre os métodos de StollHansheer e da câmara de contagem segundo Santos Ferreira. Rev. paul. Med., 74:331-2, 1969.

8. LAYRISSE, M. \& MARTINEZ-TORRES C. Model for measuring dietary absorption of heme iron test with it complete meal. Amer. J. clin. Nutr.. 25:401-11, 1972.
9. LAYRISSE, M. \& ROCHE, M. Reabsorption of hemoglobin iron lost into the intestins in hookworm-infested patients. Conference of malabsorption and allied hematological problems. Amer. J. dig. Dis., 7:976-7, 1962.

10. LAYRISSE, M, et al. Food iron absorption: a comparison of vegetables and animal foods. Blood, 33:430.43, 1969.

11. MASPES, V. et al. Contribución al estudio del metabolismo del hierro en hemopatias diversas mediante el empleo de los isotopos radiactivos ${ }^{59} \mathrm{Fe}$ y ${ }^{51} \mathrm{Cr}$. Sangre, 4:351-412, 1959.

12. MASPES, V. \& TAMIGAKI, M. Padronização de métodos para dosagem do ferro e da siderofilina no soro. Rev. Hosp. Clin., 30:126-31, 1975.

13. ROCHE, M. \& PAREZ-GIMENEZ, M. E. Intestinal loss and reabsorption of iron in hookworm infection. $J$, Lab. clin. Med., 54:49-52, 1959.

14. ROCHE, M. et al. Gastrointestinal bleeding in hookworm infection. Amer. J. dig. Dis., 2:265-77, 1957.

15. ROCHE, M. et al. Isotopic tracer method for measurement of iron lost and reabsorbed from gastrointestinal bleeding lesions. Nature, $180: 1278-9$, 1957.

Recebido para publicação em 29/06/1979 Aprovado para publicą̧āo em 30/07/197y 